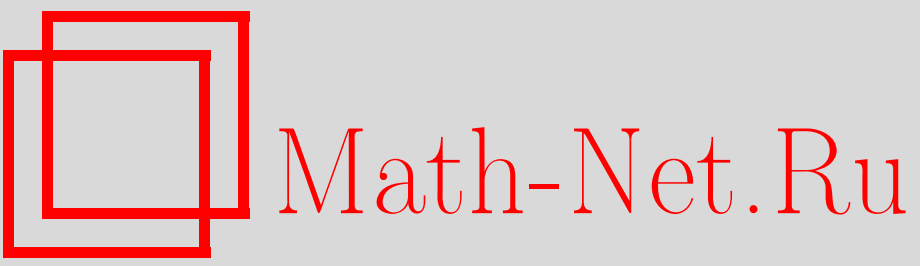

А. М. Райгородский, Об одной оценке в проблеме Борсука, УМН, 1999, том 54, выпуск 2, 185-186

DOI: https://doi.org/10.4213/rm146

Использование Общероссийского математического портала Math-Net.Ru подразумевает, что вы прочитали и согласны с пользовательским соглашением

http://www.mathnet.ru/rus/agreement

Параметры загрузки:

IP: 52.6 .47 .48

26 апреля 2023 г., $14: 03: 30$ 


\title{
ОБ ОДНОЙ ОЦЕНКЕ В ПРОБЛЕМЕ БОРСУКА
}

\author{
А. М. РАЙГОРОДСКИЙ
}

1. Введение. В [1] Борсук высказал гипотезу о том, что всякое ограниченное множество в $\mathbb{R}^{d}$ может быть разбито на $d+1$ часть меньшего диаметра. Утверждение гипотезы Борсука было доказано в размерностях 2 и 3 , а также в произвольной размерности для множеств $\mathrm{c}$ гладкой границей. В работе [2] с помощью результатов работы [3] был получен контрпример к гипотезе Борсука в размерности 1325. В [4] контрпример был построен в размерности $d=946$. В [5], за счет уточнения техники, предложенной в работе [4], удалось привести контрпример в размерности $d=561$. Из доказательства, приведенного в [5], следовало, что гипотеза Борсука может быть опровергнута при всех $d$ таких, что $561 \leqslant d \leqslant 757$ и $d \geqslant 861$. В настоящей работе мы докажем следующее

УтвеРЖДЕНИЕ. При всех $d \geqslant 561$ найдется ограниченное $d$ - мерное множество, которое нельзя разбить на $d+1$ часть меньиего диаметра

Определим величину $f(d)$ как минимальное число такое, что всякое ограниченное множество в $\mathbb{R}^{d}$ может быть разбито на $f(d)$ частей меньшего диаметра. В [6] для этой величины была получена оценка $f(d) \leqslant 2^{d-1}+1$, а в [7] было доказано, что для всякого $\varepsilon>0$ при всех достаточно больших $d: f(d) \leqslant(\sqrt{3 / 2}+\varepsilon)^{d}$. Другое доказательство последнего результата было дано в работе [8]. В [2] было показано, что $f(d) \geqslant(P(d))^{-1}(1.203)^{\sqrt{d}}$, где $P(d)$ - полином. В настоящей работе мы докажем следуюшую теорему.

Теорема. Пусть $\vartheta=(2 / \sqrt{3})^{\sqrt{2}}=1.2255895 \ldots$, mогда $f(d) \geqslant R(d) \vartheta^{\sqrt{d}}$, где $R(d)-$ такая функчия, что $R_{1}(d) \leqslant R(d) \leqslant R_{2}(d)$, причем при $d \rightarrow \infty: R_{1}(d) c^{\sqrt{d}} \rightarrow \infty u$ $R_{2}(d) / c^{\sqrt{d}} \rightarrow 0 \quad \forall c>1$.

2. Доказательство теоремы. Пусть $n \in \mathbb{N}$ - достаточно велико, $\delta=1-1 / \sqrt{3}$, а $p$ ближайшее к величине $\delta n$ нечетное простое число. Известно (см. [9]), что для достаточно большого $x$ и для некоторого $\alpha<1$ (например, для $\alpha=38 / 61$ ) между $x$ и $x+x^{\alpha}$ лежит простое число. Поэтому можно считать, что $\delta n-(\delta n)^{\alpha}<p<\delta n+(\delta n)^{\alpha}$. Заметим, что выбор величины $\alpha$ влияет исключительно на оценку функции $R(d)$. Рассмотрим семейство векторов $\Sigma \subset \mathbb{R}^{n}$ такое, что всякий вектор $\mathbf{x} \in \Sigma$ содержит $n-p$ нулевых компонент и $p$ компонент вида $x_{i_{1}}=1, x_{i_{2}}= \pm 1, \ldots, x_{i_{p}}= \pm 1\left(1 \leqslant i_{1}<i_{2}<\cdots<i_{p} \leqslant n\right)$. Таким образом, conv $\Sigma$ есть кросс-политоп, вершинами которого являются центры граней размерности $n-p$ куба $[-1,1]^{n}$. В то же время в работах [2], [4], [5] для построения контрпримеров и получения оценок величины $f(d)$ рассматривались некоторые специальные подмножества множества вершин куба $[-1,1]^{n}$. Мы покажем далее, что стандартные методы позволяют работать с кросс-политопами и на их основе получать лучшие оценки для $f(d)$. В самом деле, ясно, что card $\Sigma=2^{p-1} C_{n}^{p}$. Кроме того, легко видеть, что для любых различных векторов $\mathbf{a}, \mathbf{b} \in \Sigma$ выполнено следующее простое утверждение:

$$
(\mathbf{a}, \mathbf{b}) \equiv 0(\bmod p) \text { тогда и только тогда, когда }(\mathbf{a}, \mathbf{b})=0
$$

Каждому вектору $\mathbf{a} \in \Sigma$ поставим в соответствие многочлен $F_{\mathbf{a}} \in \mathbb{Z} / p \mathbb{Z}\left[x_{1}, \ldots, x_{n}\right]$, определяемый соотношениями $F_{\mathbf{a}}(\mathbf{x})=\prod_{i=1}^{p-1}(i-(\mathbf{a}, \mathbf{x}))$ и $x_{i}^{3}=x_{i}$. Опираясь на некоторые арифметические свойства этих многочленов, нетрудно показать, что имеет место следующая

Работа выполнена при финансовой поддержке Российского фонда фундаментальных исследований (грант № 96-01-00378). 
Лемма. Каково бы ни бъло семейство векторов $Q=\left\{\mathbf{a}_{1}, \ldots, \mathbf{a}_{s}\right\} \subset \Sigma$ такое, что $\left(\mathbf{a}_{i}, \mathbf{a}_{j}\right) \not \equiv 0(\bmod p) \forall i \neq j$, для величины $s=\operatorname{card} Q$ выполнена оценка:

$$
s \leqslant \sum_{l=0}^{p-1\left[\frac{p-1-l}{2}\right]} \sum_{k=0}^{k} C_{n}^{k-1-l-2 k} .
$$

Доказательство леммы стандартно (см. [4] и [5]), поэтому в настоящей работе мы его не приводим.

Семейству векторов $\Sigma$ сопоставим семейство $n^{2}$-мерных векторов $\Sigma^{*}$ следуюшим образом. Каждому вектору $\mathbf{x} \in \Sigma$ поставим в соответствие вектор $\mathbf{x} * \mathbf{x}=\left(x_{i} \times x_{j}\right), i=1, \ldots, n$, $j=1, \ldots, n$. Легко убедиться в том, что для любых двух векторов $\mathbf{x} * \mathbf{x}, \mathbf{z} * \mathbf{z} \in \Sigma^{*}$ выполнено равенство $(\mathbf{x} * \mathbf{x}, \mathbf{z} * \mathbf{z})=(\mathbf{x}, \mathbf{z})^{2}$. Отсюда следует, что максимальное расстояние между векторами из $\Sigma^{*}$ достигается в том и только том случае, когда скалярное произведение их прообразов равно нулю. Воспользовавшись последним рассуждением, утверждением (1) и неравенством (2), получаем, что множество $\Sigma^{*}$ невозможно разбить на $f<2^{p-1} C_{n}^{p} /\left(\sum_{l=0}^{p-1} \sum_{k=0}^{\left[\frac{p-1-l}{2}\right]} C_{n}^{k} C_{n-k}^{p-1-l-2 k}\right)$ частей так, чтобы диаметр каждой части был меньше диаметра всего множества. Кроме того понятно, что размерность семейства векторов $\Sigma^{*}$ равна $\frac{n(n+1)}{2}-1$. Положим $d=\frac{n(n+1)}{2}-1$. Из сказанного выше следует, что $f(d) \geqslant 2^{p-1} C_{n}^{p} /\left(\sum_{l=0}^{p-1} \sum_{k=0}^{\left[\frac{p-1-l}{2}\right]} C_{n}^{k} C_{n-k}^{p-1-l-2 k}\right)$. Учитывая выбор величины $p$ и проведя несложные выкладки, можно показать, что тем самым $f(d) \geqslant$ $R(d) \vartheta^{\sqrt{d}}$, и теорема доказана.

3. Доказательство утверждения. Положим $n=36, d_{0}=561$. Пусть $\Sigma^{*}=\{\mathbf{x}=$ $\left.\left(x_{1}, \ldots, x_{(n-1)(n-3)}\right)\right\}$ - семейство векторов, построенное в работе [5]. Тогда из [5] следует, что найдутся индексы $i_{1}, \ldots, i_{n-3}$ такие, что $x_{i_{j}}=1 \forall j=1, \ldots, n-3$ и $\forall \mathbf{x} \in \Sigma^{*}$. Фиксируем произвольное $d \geqslant 564$ и рассмотрим $t=d-d_{0}$ и $m=\sqrt{(n-1)(n-3)-3}+1$. Определим семейство векторов $\Sigma_{d}=\left\{\left(x_{1}, \ldots, x_{(n-1)(n-3)+t}\right)\right\}$. (Здесь $x_{i_{1}}=x_{i_{2}}=x_{i_{3}}=1$, для некоторого $2 \leqslant i \leqslant t-1: x_{(n-1)(n-3)+i}=m, x_{(n-1)(n-3)+1}=\cdots=x_{(n-1)(n-3)+i-1}=$ $x_{(n-1)(n-3)+i+1}=\cdots=x_{(n-1)(n-3)+t}=1$, а все остальные компоненты равны нулю.) Определим также $\widetilde{\Sigma}_{d}=\left\{\left(x_{1}, \ldots, x_{(n-1)(n-3)}, 1, \ldots, 1\right)\right\} \subset \mathbb{R}^{(n-1)(n-3)+t}$ как семейство векторов, полученных из векторов семейства $\Sigma^{*}$ добавлением $t$ компонент, равных единице. Рассмотрим наконец семейство векторов $\Sigma_{d}^{*}=\Sigma_{d} \sqcup \widetilde{\Sigma}_{d}$. Нетрудно видеть, основываясь на результатах работы [5], что размерность линейного подпространства, в котором лежит $\Sigma_{d}^{*}$, равна $d_{0}+1+$ $(t-2)=d-1$. Кроме того, из [5] следует, что диаметр множества $\widetilde{\Sigma}_{d}$ равен $\sqrt{2(n-1)(n-3)-6}$. В то же время расстояние между любыми двумя векторами из $\Sigma_{d}$, а также между любыми двумя векторами, один из которых принадлежит $\Sigma_{d}$, а другой $\widetilde{\Sigma}_{d}$, равно $\sqrt{2(n-1)(n-3)-6}$. Из этого факта и оценки, полученной в [5], следует, что $f(d-1) \geqslant f\left(d_{0}\right)+t-2 \geqslant 755+t>d$. При $d=562$ контрпример фактически построен в [5]. Утверждение доказано.

\section{СПИСОК ЛИТЕРАТУРЫ}

[1] Borsuk K. // Fund. Math. 1933. V. 20. P. 177-190. [2] Kahn J., Kalai G. // Bull. Amer. Math. Soc. 1993. V. 29. №1. P. 60-62. [3] Frankl P., Wilson R. // Combinatorica. 1981. V. 1. P. 259-286. [4] Nilli A. // Contemp. Math. 1994. V. 178. Р. 209-210. [5] Райгородский A. M. // УMH. 1997. T. 52. №6. C. 181-182. [6] Lassak M. // Bull. Polish Acad. Sci. Math. 1982. V. 30. P. 449-451. [7] Schramm O. // Mathematika. 1988. V. 35. P. 180-199. [8] Bourgain J., Lindenstrauss J. // Lecture Notes in Math. 1991. V. 1469. Р. 138-144. [9] Пpaxap K. Pacпределение простых чисел. М.: Мир, 1967. 